\section{Forward Gated-Diode Measurement of Filled Traps in High-Field Stressed Thin Oxides}

Ming-Jer Chen, Ting-Kuo Kang, Huan-Tsung Huang, Chuan-Hsi Liu, Yih J. Chang, and Kuan-Yu Fu

\begin{abstract}
The forward gated-diode monitoring technique can find its potential applications in assessing the filled traps in MOSFET thin oxides, which are subjected to high-field stressing and then followed by hot-electrons filling scheme. Our measurement of the gate voltage shift associated with the forward current peak produces a power law relation between the filled trap density and the electron stress fluence, indeed in close agreement with that obtained by MOSFET threshold voltage shift.
\end{abstract}

Index Terms-Gated-diode, hot electron, MOSFET, neutral trap, oxide breakdown, SILC, thin oxide.

\section{INTRODUCTION}

The high-field Fowler-Nordheim (FN) electron tunneling through thin oxides can produce a variety of defects, among which the most concerned are the neutral electron traps. The principal reasons are that 1) the neutral electron traps can serve as a stepping stone for injected electrons, which gives rise to SILC in low voltage regime [1],[2], and 2) a certain trap density is critically encountered, leading to a breakdown event [3]-[6]. Thus, an essential knowledge of the total neutral trap density $N_{T}$ created for imposed electron fluence $Q_{e}$ is crucial to the study of SILC and oxide breakdown. To achieve this goal, Degraeve et al. [7], [8] have recently performed two independent experiments while introducing a key physical parameter, namely, filling or occupied fraction $p$ [9], to connect the two. The first experiment is the hot-electrons filling scheme following the high-field stress. This scheme via a back-gate reverse bias can offer hot substrate electrons to climb over the $\mathrm{Si} / \mathrm{SiO}_{2}$ barrier height and fill the neutral traps within the oxide. Measurement of a saturation level in threshold voltage shift can be directly linked to the filled trap density $N_{o x}$, systematically leading to a power law relation [7], [8]: $N_{o x} \propto Q_{e}^{0.56 \sim 0.6}$. The second is the sphere-based Monte Carlo percolation simulation experiment treating $N_{T}$ as well as its statistics. $p$ is $N_{o x}$ divided by $N_{T}$ [9] and can be estimated by subsequently fitting intrinsic charge-to-breakdown data in the manufacturing processes [7], [8], [10], [11].

On the other hand, for MOSFET's biased in a reverse gated-diode mode [12], measurement of the reverse current $I_{r}$ versus gate voltage from accumulation through depletion to inversion can provide information concerning interface states and/or oxide traps. This mode is usually insensitive to oxides having small areas as in miniaturized devices, and the large-area oxides are inevitably required. The same information can be substantially maintained for switching to the forward mode as reflected by a well-defined relation $I_{f}=I_{r} \exp \left(q V_{f} / 2 K T\right)$ [12], where $I_{f}$ is the forward current measured at a forward bias $V_{f}$. Thus, operating in forward mode can not only make the gated-diode monitoring exponentially sensitive but also allow use of small-area oxides. Indeed, the forward gated-diode configuration formed on the stressed

Manuscript received December 2, 1999. This work was supported by the National Science Council under Contract 89-2215-009-049. The review of this brief was arranged by Editor C.-Y. Lu.

M.-J. Chen, T.-K. Kang, and H.-T. Huang are with the Department of Electronics Engineering, National Chiao-Tung University, Hsinchu, Taiwan, R.O.C. (e-mail: mjchen@RPL.ee.nctu.edu.tw).

C.-H. Liu, Y. J. Chang, and K.-Y. Fu are with United Microelectronics Corporation, Hsinchu, Taiwan, R.O.C.

Publisher Item Identifier S 0018-9383(00)06050-0.

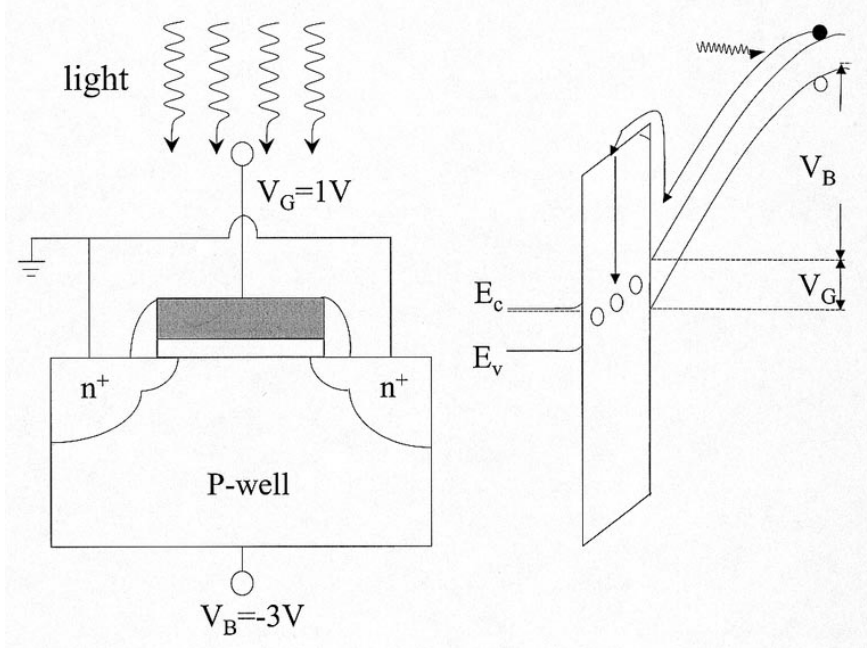

Fig. 1. Experimental setup and energy band diagram to schematically demonstrate the optically induced hot-electrons filling scheme.

MOSFET's has exhibited these abilities [13]-[15]. This work is to extend such sensitive technique to the case of thin oxides that are subjected to FN tunneling stress and then followed by hot-electrons filling scheme. The ultimate objective is to build a power law relation between $N_{o x}$ and $Q_{e}$ as that in [7], [8].

\section{EXPERIMENTAL}

The n-channel MOSFET's under study had the gate width-to-length ratio of $20 \mu \mathrm{m} / 0.3 \mu \mathrm{m}$ and the gate oxide thickness of $7 \mathrm{~nm}$. The FN tunneling stress condition was carried out at the oxide field strength $E_{o x}$ of $9.9 \mathrm{MV} / \mathrm{cm}$ with the source, drain, and substrate tied to ground, then followed by the optically induced hot- electrons filling scheme [16]. Fig. 1 shows schematically this scheme in terms of 1) the photogeneration technique via a tungsten lamp to produce electron seed in substrate, and 2) a negative back-gate bias of $-3 \mathrm{~V}$ to make substrate electrons hot such as to surmount the $\mathrm{Si} / \mathrm{SiO}_{2}$ barrier height and fill the traps. During measuring the gated-diode forward current in the drain, the drain was connected to $-0.2 \mathrm{~V}$ bias, the substrate was tied to ground, and the source was kept open.

Fig. 2 plots the measured forward current versus gate voltage for $Q_{e}=0.22 \mathrm{C} / \mathrm{cm}^{2}$ with filling or illumination time as parameter. It can be observed that the current peak in depletion region shifts toward the positive gate voltage for increasing illumination time and gradually tends to saturate. Fig. 3 shows the corresponding voltage shift $\Delta V_{G}$ associated with the current peak versus illumination time. Detrapping and Coulombic repulsion [9], which limit only part of the neutral traps available for filling, are responsible for the saturating behavior in Fig. 3. Assuming that the occupied traps are distributed uniformly within the oxide as adopted elsewhere [7], [8], [16], the saturated voltage shift $\Delta V_{G(s a t)}$ can be directly linked to the filled trap density through $\Delta V_{G(s a t)}=q t_{o x}^{2} N_{o x} / 2 \varepsilon_{o x}$ where $t_{o x}$ is the oxide thickness and $\varepsilon_{o x}$ is the oxide permittivity. The resulting $N_{o x}$ for different $Q_{e}$ is depicted in the inset of Fig. 3, showing a power law relation

$$
N_{o x}=\eta Q_{e}^{0.5}
$$

where $\eta=1.62 \times 10^{18} \mathrm{~cm}^{-2} C^{-0.5}$ for $N_{o x}$ in $1 / \mathrm{cm}^{3}$ and $Q_{e}$ in $\mathrm{C} / \mathrm{cm}^{2}$. The carrier separation technique has measured the substrate hole current, yielding hole generation coefficient of $6.8 \times 10^{-4}$ at the same stress field $E_{o x}$ of $9.9 \mathrm{MV} / \mathrm{cm}$. Thus, we have $N_{o x}=6.21 \times$ 


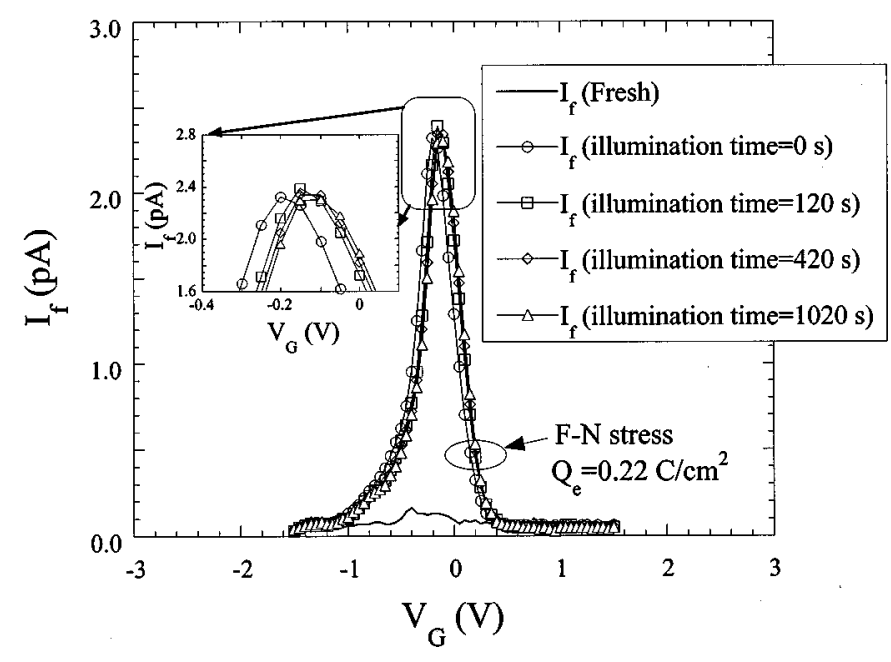

Fig. 2. Measured forward gated-diode current versus gate voltage from one n-MOSFET sample. The gate oxide area was $3 \mu \mathrm{m}^{2}$. For the selected illumination time following $Q_{e}$ of $0.22 \mathrm{C} / \mathrm{cm}^{2}$, the filling scheme was interrupted and the test setup was switched to the forward gated-diode configuration. The $I-V$ near the current peak is magnified for clear viewing.

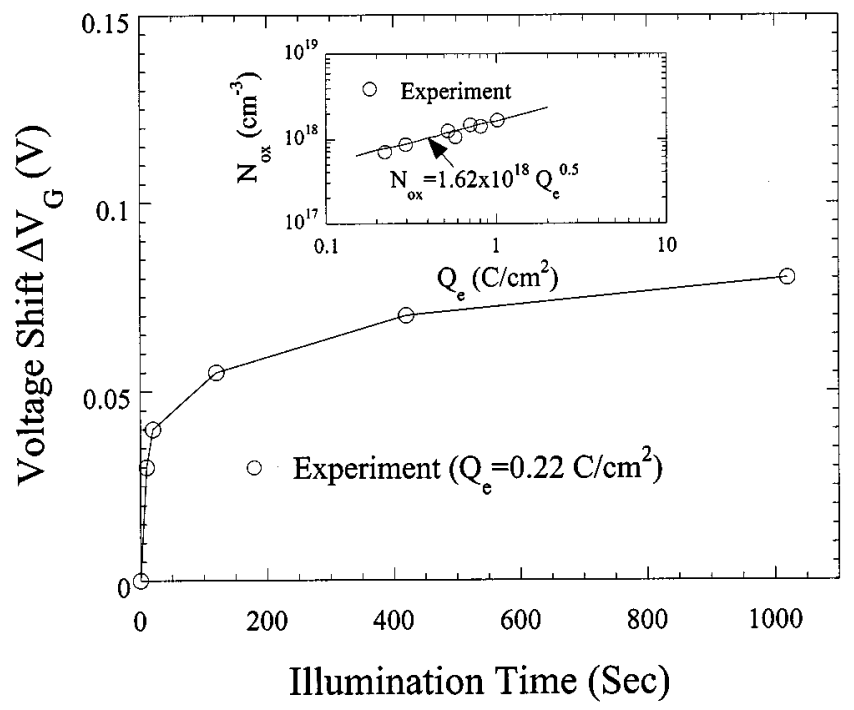

Fig. 3. Gate voltage shift versus illumination time corresponding to Fig. 2. The inset shows the estimated trap density $N_{o x}$ versus $Q_{e}$ from seven n-MOSFET samples. Each sample represents a specific $Q_{\epsilon}$. A power law relation is drawn by best fitting data points.

$10^{19} Q_{p}^{0.5}$ for hole fluence $Q_{p}$ in $\mathrm{C} / \mathrm{cm}^{2}$, which is quite close to the published expression $N_{o x}=5.3 \times 10^{19} Q_{p}^{0.56}$ [7], [8] with respect to the power exponent and the prefactor.

The above hot-electrons filling scheme has also been performed on the fresh devices, evidencing no noticeable voltage shift in the measured forward current versus gate voltage before and after the scheme.
This means that no extra neutral traps can be generated singly due to filling action. Additionally, Fig. 2 clearly reveals that the current peaks for different illumination times following FN stress (including the zero illumination time) are almost unchanged, indicating that no significant interface states can be created under the influence of the illumination induced hot electrons.

\section{CONCLUSION}

The forward gated-diode technique has demonstrated its new merit of producing a power law relation between the filled trap density and the electron stress fluence. This relationship is found to agree closely with that obtained by MOSFET threshold voltage shift.

\section{REFERENCES}

[1] D. J. Dumin and J. R. Maddux, "Correlation of stress-induced leakage current in thin oxides with trap generation inside the oxides," IEEE Trans. Electron Devices, vol. 40, pp. 986-993, 1993.

[2] D. J. DiMaria and E. Cartier, "Mechanism for stress-induced leakage currents in thin silicon dioxide films," J. Appl. Phys., vol. 78, pp. 3883-3894, 1995.

[3] E. Avni and J. Shappir, "A model for silicon-oxide breakdown under high field and current stress," J. Appl. Phys., vol. 64, pp. 743-748, 1988.

[4] J. Sune, I. Placencia, N. Barniol, E. Farres, F. Martin, and X. Aymerich, "On the breakdown statistics of very thin $\mathrm{SiO}_{2}$ films," Thin Solid Films, vol. 185 , pp. 347-362, 1990

[5] D. J. Dumin, J. R. Maddux, R. S. Scott, and R. Subramoniam, "A model relating wearout to breakdown in thin oxides," IEEE Trans. Electron Devices, vol. 41, pp. 1570-1580, 1994.

[6] P. P. Apte and K. C. Saraswat, "Modeling ultrathin dielectric breakdown on correlation of charge trap-generation to charge-to-breakdown," in Proc. IEEE IRPS, 1994, pp. 136-142.

[7] R. Degraeve et al., "A consistent model for the thickness dependence of intrinsic breakdown in ultrathin oxides," IEDM Tech. Dig., pp. 863-866, 1995.

[8] R. Degraeve et al., "New insights in the relation between electron trap generation and the statistical properties of oxide breakdown," IEEE Trans. Electron Devices, vol. 45, pp. 904-911, 1998.

[9] Y. Nissan-Cohen, J. Shappir, and D. Frohman-Bentchkowsky, "Trap generation and occupation dynamics in $\mathrm{SiO}_{2}$ under charge injection stress," J. Appl. Phys., vol. 60, pp. 2024-2035, 1986.

[10] H. T. Huang et al., "A trap generation statistical model in closed-form for intrinsic breakdown of ultrathin oxides," in Proc. IEEE Int. Symp. VLSI-TSA, 1999, pp. 70-73.

[11] M. J. Chen et al., "Cell-based analytic statistical model with correlated parameters for intrinsic breakdown of ultrathin oxides," IEEE Electron Device Lett., vol. 20, pp. 523-525, 1999.

[12] A. S. Grove, Physics and Technology of Semiconductor Devices. New York: Wiley, 1967, ch. 10.

[13] A. Acovic, M. Dutoit, and M. Ilegems, "Characterization of hot-electron-stressed MOSFET's by low-temperature measurements of the drain tunnel current," IEEE Trans. Electron Devices, vol. 37, pp. 1467-1476, 1990.

[14] P. Speckbacher et al., "The "gated-diode" configuration in MOSFET's, a sensitive tool for characterizing hot-carrier degradation," IEEE Trans. Electron Devices, vol. 42, pp. 1287-1296, 1995.

[15] C. H. Ling and Y. H. Goh, "Close correspondence between forward gated-diode and charge pumping currents observed in hot-carrier stressed PMOSFET's," IEEE Trans. Electron Devices, vol. 44, pp. 2309-2311, 1997.

[16] T. H. Ning, C. M. Osburn, and H. N. Yu, "Emission probability of hot electrons from silicon into silicon dioxide," J. Appl. Phys., vol. 48, pp. 286-293, 1977. 